\title{
Recent progress in the effective string theory description of LGTs.
}

\section{Marco Billò $^{a}$, Michele Caselle ${ }^{* a}$, Davide Fioravanti ${ }^{c}$, Ferdinando Gliozzi ${ }^{a, b}$, Marco Meineri $^{d}$, Roberto Pellegrini ${ }^{a}$, Roberto Tateo ${ }^{a}$}

a Dipartimento di Fisica, Università di Torino

and Istituto Nazionale di Fisica Nucleare, sezione di Torino

via P. Giuria 1, 10125 Torino (Italy)

${ }^{b}$ School of Computing and Mathematics \& Centre for Mathematical Science,

Plymouth University, Plymouth PL4 8AA, UK

${ }^{c}$ Istituto Nazionale di Fisica Nucleare - sezione di Bologna

and Dipartimento di Fisica e Astronomia, Università di Bologna,

Via Irnerio 46, I- 40127 Bologna, Italy

${ }^{d}$ Scuola Normale Superiore, Piazza dei Cavalieri 7 I-56126 Pisa, Italy

and Istituto Nazionale di Fisica Nucleare - sezione di Pisa

E-mail: (billo) (caselle) (gliozzi) (ropelleg) (tateo) dto.infn.it

fioravantiabo.infn.it, marco.meineriesns.it

In presence of a static pair of sources, the spectrum of low-lying states of any confining gauge theory in D space-time dimensions is described, at large source separations, by an effective string theory. Recently two important advances improved our understanding of this effective theory. First, it was realized that the form of the effective action is strongly constrained by the requirement of the Lorentz invariance of the gauge theory, which is spontaneously broken by the formation of a long confining flux tube in the vacuum. This constraint is strong enough to fix uniquely the first few subleading terms of the action. Second, it has been realized that the first of these allowed terms - a quartic polynomial in the field derivatives - is exactly the composite field $T \bar{T}$, built with the chiral components, $T$ and $\bar{T}$, of the energy-momentum tensor of the 2d QFT describing the infrared limit of the effective string. This irrelevant perturbation is quantum integrable and yields, through the thermodynamic Bethe Ansatz (TBA), the energy levels of the string which exactly coincide with the Nambu-Goto spectrum. In this talk we first review the general implications of these two results and then, as a test of the power of these methods, use them to construct the first few boundary corrections to the effective string action.

31st International Symposium on Lattice Field Theory - LATTICE 2013

July 29 - August 3, 2013

Mainz, Germany

\footnotetext{
* Speaker.
} 


\section{Introduction}

It is widely believed that the dynamics of the flux tube joining a quark-antiquark pair is well described by an effective string action $S$ which flows at large scales towards a massless free-field theory. Thus, for large enough inter-quark separations it is not necessary to know explicitly the specific form of the effective string action $S$, but only its infrared limit [1]

$$
S[X]=S_{c l}+S_{0}[X]+\ldots,
$$

where the classical action $S_{c l}$ describes the usual perimeter-area term, $X$ denotes the $2 \mathrm{~d}$ bosonic fields $X_{i}\left(\xi_{1}, \xi_{2}\right)$, with $i=1,2, \ldots, D-2$, describing the transverse displacements of the string with respect the configuration of minimal energy, $\xi_{1}, \xi_{2}$ are the coordinates on the world-sheet and $S_{0}[X]$ is the Gaussian action

$$
S_{0}[X]=\frac{\sigma}{2} \int d^{2} \xi\left(\partial_{\alpha} X \cdot \partial^{\alpha} X\right)
$$

In the past few years there has been substantial progress in our understanding of the effective string action both from the theoretical and from the numerical point of view. In particular we want to discuss in this short review two major advances:

- The Effective String action is strongly constrained by Lorentz invariance. The first few corrections in eq. (1.1) are uniquely fixed and coincide with those arising from the NambuGoto action. This explains why Nambu-Goto describes so well the infrared regime of Wilson loops or Polyakov loop correlators.

- The Nambu-Goto effective string can be described as a free $2 \mathrm{~d}$ bosonic theory perturbed by the irrelevant operator $T \bar{T}$ (where $T$ and $\bar{T}$ are the two chiral components of the energy momentum tensor). This perturbation is quantum integrable and yields, using the Thermodynamic Bethe Ansatz (TBA), a spectrum which, in a suitable limit, coincides with the Nambu-Goto one.

As an example of the possible applications of these results in the final part of this paper we shall use them to study the boundary corrections to the effective string action and evaluate their contribution to Wilson loops and Polyakov loop correlators. We shall then test these predictions with high precision numerical estimates in the $3 \mathrm{D}$ gauge Ising model.

\section{Lorentz invariance}

The Effective String action may contain only terms respecting the internal and space-time symmetries of the system. It can be written as a low energy expansion in the number of derivatives of the $X$ fields. In particular the first few terms beyond the free $2 \mathrm{~d}$ bosonic theory of eq. (1.2) for an open string stretched between fixed ends, for instance Polyakov loops, are [2]

$$
S=S_{c l}+\frac{\sigma}{2} \int d^{2} \xi\left[\partial_{\alpha} X \cdot \partial^{\alpha} X+c_{2}\left(\partial_{\alpha} X \cdot \partial^{\alpha} X\right)^{2}+c_{3}\left(\partial_{\alpha} X \cdot \partial_{\beta} X\right)^{2}+\ldots\right]+S_{b},
$$


where $S_{b}$ is the boundary contribution characterizing the open string. If the boundary is a Polyakov line in the $\xi_{0}$ direction placed at $\xi_{1}=0$, on which we assume Dirichlet boundary conditions $X_{i}\left(\xi_{0}, 0\right)=0$, the first few terms contributing to $S_{b}$ are

$$
S_{b}=\int d \xi_{0}\left[b_{1} \partial_{1} X \cdot \partial_{1} X+b_{2} \partial_{1} \partial_{0} X \cdot \partial_{1} \partial_{0} X+b_{3}\left(\partial_{1} X \cdot \partial_{1} X\right)^{2}+\ldots\right] .
$$

As first observed in 2004 by Lüscher and Weisz [2] the coefficients $c_{i}$ and $b_{i}$ of the above expansion should satisfy some consistency constraints; they were obtained by the comparison of the string partition function in different channels ("open-closed string duality"). These results were further generalized in [3]. However it was later realized [4, 5] that the crucial ingredient of these constraints is the Lorentz symmetry of the underlying Yang-Mills theory. Indeed, even if the complete $S O(1, D-1)$ invariance is broken by the classical configuration around which we expand, the effective action should still respect this symmetry through a non-linear realisation in terms of the transverse fields $X_{i}$. In this way it was shown $[5,6]$ that the terms with only first derivatives coincide with the Nambu-Goto action to all orders in the derivative expansion. The first allowed correction to the Nambu-Goto action in the bulk turns out to be the the six derivative term [3]

$$
c_{4}\left(\partial_{\alpha} \partial_{\beta} X \cdot \partial^{\alpha} \partial^{\beta} X\right)\left(\partial_{\gamma} X \cdot \partial^{\gamma} X\right)
$$

with arbitrary coefficient $c_{4}$; however this term is non-trivial only for $D>3$. At $D=3$ the first non-trivial deviation to the Nambu-Goto action is an eight-derivative term and it has been recently shown [7], using the recursion relations generated by the non-linear Lorentz transformations, that it generates a geometric term proportional to the squared curvature of the induced metric on the world-sheet. These recursion relations and their relationship with diffeomorphism invariance were further investigated in [8,9], while the link with the orthogonal gauge (Polchinski-Strominger) approach to the effective action was studied in [10] (see also [11]). The fact that the first deviations from the Nambu-Goto string are of high order, especially in $D=3$, explains why in early Monte Carlo calculations $[12,13,14]$ a good agreement with the Nambu-Goto string was observed. A similar analysis can be performed for the the effective string spectrum [15]. Also in this case, for the majority of the states, a good agreement with lattice simulations was found $[16,17]$.

\section{The Nambu-Goto action as the $T \bar{T}$ perturbation of $S_{0}$.}

The energy-momentum tensor of the free-field theory (1.2) can be written as

$$
T_{\alpha \beta}=\partial_{\alpha} X \cdot \partial_{\beta} X-\frac{1}{2} \delta_{\alpha \beta}\left(\partial^{\gamma} X \cdot \partial_{\gamma} X\right) .
$$

Setting in eq. (2.1) the values $c_{2}=\frac{1}{8}$ and $c_{3}=-\frac{1}{4}$ prescribed by Lorentz invariance, we find

$$
S=S_{c l}+S_{0}[X]-\frac{\sigma}{4} \int d^{2} \xi T_{\alpha \beta} T^{\alpha \beta}+S_{b}+\ldots
$$

In $2 \mathrm{~d}$ CFTs it is useful to introduce the chiral components $T_{z z}=\frac{1}{2}\left(T_{11}-i T_{12}\right)$ and $T_{\bar{z} \bar{z}}=\frac{1}{2}\left(T_{11}+i T_{12}\right)$ and use the normalized quantities $T=-2 \pi \sigma T_{z z}, \bar{T}=-2 \pi \sigma T_{\bar{z} z}$ in such a way the operator product expansion begins with

$$
T(z) T(w)=\frac{D-2}{2} \frac{1}{(z-w)^{4}}+\ldots
$$


and similarly for $\bar{T}$. Thus we may write

$$
S=S_{c l}+S_{0}[X]-\frac{1}{2 \pi^{2} \sigma} \int d^{2} \xi T \bar{T}+S_{b}+\ldots
$$

This shows that the (universal) effective string theory at the next to leading order is nothing else than the $T \bar{T}$ perturbation of the free field theory [20]. This massless perturbation can be studied using TBA techniques. For the ground state with periodic boundary conditions, this was pioneered several years ago by Zamolodchikov [18]. Let us briefly review the TBA analysis of [18, 19, 20]. The simplest instance discussed in [18] corresponds to the RG flow connecting the tricritical Ising to the Ising model and it is described by a single species of interacting massless Majorana fermions. Close to the IR fixed point the model is described by an effective action of the form (3.4) with $S_{b}=0$ :

$$
S=S_{\text {Ising }}-\frac{1}{2 \pi^{2} \sigma} \int d^{2} \xi T \bar{T}+\ldots,(\sigma \gg 1),
$$

where $\sigma$ sets the scale of the perturbation and will play the role of string tension in our effective string interpretation. In 1+1-dimensions, massless excitations confined on a ring naturally separate into right and left movers. In the case under consideration, the right-right and left-left mover scattering is trivial while the left-right scattering is described by the amplitude

$$
S(p, q)=\frac{2 \sigma+i p q}{2 \sigma-i p q},
$$

where $p$ is the momentum of the right mover and $-q$ the momentum of the left mover. Starting from the $S$-matrix (3.6), Zamolodchikov was able to derive the TBA equations for the vacuum energy of the theory defined on a infinite cylinder with circumference $R$. Considering also the excited states $[21,22]$, the TBA equations are

$$
\varepsilon(p)=R p-\int_{\overline{\mathscr{C}}} \frac{d q}{2 \pi} \phi(p, q) \bar{L}(q), \quad \bar{\varepsilon}(p)=R p-\int_{\mathscr{C}} \frac{d q}{2 \pi} \phi(p, q) L(q),
$$

where $\varepsilon(p)$ and $\bar{\varepsilon}(p)$ are the pseudoenergies for the right and the left movers, respectively, and

$$
\phi(p, q)=-i \partial_{q} \ln S(p, q), L(p)=\ln \left(1+e^{-\varepsilon(p)}\right), \bar{L}(p)=\ln \left(1+e^{-\bar{\varepsilon}(p)}\right) .
$$

In (3.7), the integration contours $\mathscr{C}$ and $\overline{\mathscr{C}}$ run from $q=0$ to $q=\infty$ on the real axis for the ground state, but for excited states they circle around a finite number of poles $\left\{q_{i}\right\}$ and $\left\{\bar{q}_{i}\right\}$ of $\partial_{q} L(q)$ and $\partial_{q} \bar{L}(q)$ (cf. $[19,20]$ ). The corresponding energy is

$$
E^{(T B A)}(R, \sigma)=-\int_{\mathscr{C}} \frac{d p}{2 \pi} L(p)-\int_{\overline{\mathscr{C}}} \frac{d p}{2 \pi} \bar{L}(p) .
$$

From (3.7-3.9), the $T \bar{T}$ contribution to the energies can be spotted only perturbatively [18], but if we consider only the leading part $S_{1}(p, q)$ of the Zamolodchikov's $S$ matrix at large $\sigma$

$$
S(p, q)=e^{i p q / \sigma-i(p q / \sigma)^{3} / 12+\ldots}=S_{1}(p, q) e^{-i(p q / \sigma)^{3} / 12+\ldots}
$$

then the kernel becomes $\phi(p, q)=-i \partial_{q} \ln S_{1}(p, q)=p / \sigma$, and the corresponding TBA equations can be solved explicitly [19]. In fact, it is easy to show that they are fully equivalent to the following simple algebraic equations [20]:

$$
E_{(n, \bar{n})}(R, \sigma)=E^{(T B A)}(R, \sigma)+\sigma R=\mathscr{E}+\overline{\mathscr{E}}+\sigma R,
$$




$$
\mathscr{E}=-\frac{\pi\left(\tilde{c}_{I R}-24 n\right)}{12(R+\overline{\mathscr{E}} / \sigma)}, \quad \overline{\mathscr{E}}=-\frac{\pi\left(\tilde{c}_{I R}-24 \bar{n}\right)}{12(R+\mathscr{E} / \sigma)}
$$

with $n, \bar{n} \in \mathbb{N}$. These equations can be easily solved, giving

$$
E_{(n, \bar{n})}(R, \sigma)= \pm \sqrt{\sigma^{2} R^{2}+4 \pi \sigma\left(n+\bar{n}-\frac{\tilde{c}_{I R}}{12}\right)+\left(\frac{2 \pi(n-\bar{n})}{R}\right)^{2}} .
$$

Setting $\tilde{c}_{I R}=D-2$ this becomes exactly the N-G spectrum. For the massless flow (3.5) $\tilde{c}_{I R}=$ $c_{I s i n g}=1 / 2$, while for the single boson field theory discussed in [19] $\tilde{c}_{I R}=1$. However, it is interesting to notice that the result is more general and holds not only for free field theories but also for more general infrared CFTs [20]. The only change is that $\tilde{c}_{I R}=c_{I R}-24 h$ where $h$ may be anyone of the conformal weights of the theory (and can be tuned using suitable boundary conditions).

\section{Example: the boundary term}

Using the results of the previous sections we can fix the expression of the first few terms of the boundary action eq. (2.2) and find its contribution to the effective string spectrum. It was already realized in [2] that $b_{1}=0$. It was later observed in [5] that also $b_{3}=0$ and only the $b_{2}$ term is compatible with Lorentz invariance. In [23] we completed the analysis by solving the recursion relations dictated by the requirement of Lorentz invariance. The resulting expansion can be written in the closed form

$$
b_{2} \int d \xi_{0}\left[\frac{\partial_{0} \partial_{1} X \cdot \partial_{0} \partial_{1} X}{1+\partial_{1} X \cdot \partial_{1} X}-\frac{\left(\partial_{0} \partial_{1} X \cdot \partial_{1} X\right)^{2}}{\left(1+\partial_{1} X \cdot \partial_{1} X\right)^{2}}\right] .
$$

In order to estimate the coefficient $b_{2}$ we performed a set of high precision simulations in the 3D gauge Ising model, evaluating both Wilson loops and Polyakov loop correlators. Within the precision of our data we could measure only the contribution of the first term in the expansion of eq. (4.1):

$$
S_{b, 2}^{(1)}=b_{2} \int_{\partial \Sigma}\left(\partial_{0} \partial_{1} X\right)^{2} .
$$

The contribution of this term to the interquark potential (the ground state of the effective string spectrum) depends on the geometry of the observable. In the case of Polyakov loop correlators we have [5]

$$
\left\langle S_{b, 2}^{(1)}\right\rangle_{P}=-b_{2} \frac{\pi^{3} L}{60 R^{4}} E_{4}\left(\mathrm{i} \frac{L}{2 R}\right),
$$

where $R$ is the distance between the two Polyakov loops and $L$ the length of the compactified time direction (i.e. the inverse temperature).

For the Wilson loop we have [23]

$$
\left\langle S_{b, 2}^{(1)}\right\rangle_{W}=-b_{2} \frac{\pi^{3}}{60}\left[\frac{R}{L^{4}} E_{4}\left(\mathrm{i} \frac{R}{L}\right)+\frac{L}{R^{4}} E_{4}\left(\mathrm{i} \frac{L}{R}\right)\right],
$$

where the Eisenstein function $E_{4}$ is defined as:

$$
E_{4}(\tau)=1+240 \sum_{n=1}^{\infty} \sigma_{3}(n) q^{n},
$$


where $q=\exp (2 \pi \mathrm{i} \tau)$ and $\sigma_{p}(n)$ is the sum of the $p$-th powers of the divisors of $n$ :

$$
\sigma_{p}(n)=\sum_{m \mid n} m^{p}
$$

In both cases these expressions turn out to fit remarkably well the numerical data allowing to obtain a rather precise estimate for $b_{2}$. The results for the three different values of $\beta$ that we simulated in [23] are reported in table 1.

\begin{tabular}{|cccc|}
\hline data set & $b_{2}$ & $b_{2} \sqrt{\sigma}^{3}$ & $\chi^{2}$ \\
\hline 1 & $7.25(15)$ & $0.0250(5)$ & 1.2 \\
2 & $26.8(8)$ & $0.0289(9)$ & 1.8 \\
3 & $57.9(12)$ & $0.0319(7)$ & 1.3 \\
\hline
\end{tabular}

Table 1: Values of $b_{2}$ obtained from the fits.

Looking at the third column we see that the data show a rather good scaling behaviour and allow us to propose as a final estimate for the $b_{2}$ parameter in the 3D gauge Ising model the value:

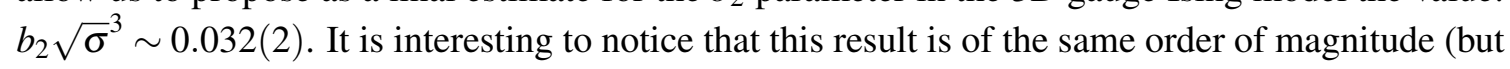
opposite in sign) of the value of $b_{2}$ measured in the 3D $S U(2)$ gauge model in [24].

\section{Acknowledgements}

M. M. was a visiting graduate fellow at the Perimeter Institute for Theoretical Physics (Waterloo, Ontario) while this work was completed. Research at Perimeter Institute is supported by the Government of Canada through Industry Canada and by the Province of Ontario through the Ministry of Economic Development and Innovation. This work is supported in part by the Compagnia di San Paolo contract "Modern Application in String Theory" (MAST) TO-Call3-2012-0088.

\section{References}

[1] M. Luscher, K. Symanzik and P. Weisz, Anomalies of the free loop wave equation in the WKB Approximation, Nucl. Phys. B173, 365 (1980).

[2] M. Luscher and P. Weisz, String excitation energies in $S U(N)$ gauge theories beyond the free-string approximation, JHEP 07, 014 (2004), [arXiv:hep-th/0406205].

[3] O. Aharony and E. Karzbrun, On the effective action of confining strings, JHEP 06, 012 (2009), [arXiv:0903.1927 [hep-th]].

[4] H. B. Meyer, Poincare invariance in effective string theories, JHEP 05, 066 (2006), [arXiv:hep-th/0602281].

[5] O. Aharony and M. Field, On the effective theory of long open strings, JHEP 1101, 065 (2011), [arXiv:1008.2636 [hep-th]].

[6] F. Gliozzi, Dirac-Born-Infeld action from spontaneous breakdown of Lorentz symmetry in brane-world scenarios, Phys. Rev. D84, 027702 (2011), [arXiv:1103.5377 [hep-th]].

[7] O. Aharony and M. Dodelson, Effective string theory and nonlinear Lorentz invariance, [arXiv:1111.5758 [hep-th]]. 
[8] F. Gliozzi and M. Meineri, Lorentz completion of effective string (and p-brane) action, JHEP 1208, 056 (2012), [arXiv:1207.2912 [hep-th]].

[9] P. Cooper, Stückelberg Fields on the Effective p-brane, Phys. Rev. D88, 025047 (2013), [arXiv:1303.0743 [hep-th]].

[10] S. Dubovsky, R. Flauger and V. Gorbenko, Effective string theory revisited, JHEP 1209, 044 (2012), [arXiv:1203.1054 [hep-th]].

[11] O. Aharony and Z. Komargodski, The effective theory of long strings, [arXiv:1302.6257 [hep-th]].

[12] M. Caselle, R. Fiore, F. Gliozzi, M. Hasenbusch and K. Pinn, et al., Rough interfaces beyond the Gaussian approximation, Nucl. Phys. B432, 590 (1994), [hep-lat/9407002 [hep-lat]].

[13] M. Caselle, M. Hasenbusch and M. Panero, Comparing the Nambu-Goto string with LGT results, JHEP 03, 026 (2005), [arXiv:hep-lat/0501027].

[14] M. Caselle, M. Hasenbusch and M. Panero, High precision Monte Carlo simulations of interfaces in the three-dimensional ising model: A comparison with the Nambu-Goto effective string model, JHEP 0603, 084 (2006), [arXiv:hep-lat/0601023 [hep-lat]].

[15] O. Aharony and N. Klinghoffer, Corrections to Nambu-Goto energy levels from the effective string action, JHEP 12, 058 (2010), [arXiv:1008.2648 [hep-th]].

[16] A. Athenodorou, B. Bringoltz and M. Teper, Closed flux tubes and their string description in D=2+1 $S U(N)$ gauge theories, JHEP 05, 042 (2011), [arXiv:1103.5854 [hep-lat]].

[17] A. Athenodorou, B. Bringoltz and M. Teper, Closed flux tubes and their string description in D=3+1 $S U(N)$ gauge theories, JHEP 02, 030 (2011), [arXiv:1007.4720 [hep-lat]].

[18] A. B. Zamolodchikov, From tricritical Ising to critical Ising by thermodynamic Bethe Ansatz, Nucl. Phys. B358, 524 (1991).

[19] S. Dubovsky, R. Flauger and V. Gorbenko, Solving the simplest theory of quantum gravity, JHEP 1209, 133 (2012), [arXiv:1205.6805 [hep-th]].

[20] M. Caselle, D. Fioravanti, F. Gliozzi and R. Tateo, Quantisation of the effective string with TBA, JHEP 1307, 071 (2013), [arXiv:1305.1278 [hep-th]].

[21] V. V. Bazhanov, S. L. Lukyanov and A. B. Zamolodchikov, Integrable quantum field theories in finite volume: Excited state energies, Nucl. Phys. B489, 487 (1997), [hep-th/9607099].

[22] P. Dorey and R. Tateo, Excited states by analytic continuation of TBA equations, Nucl. Phys. B482, 639 (1996), [hep-th/9607167].

[23] M. Billo, M. Caselle, F. Gliozzi, M. Meineri and R. Pellegrini, The Lorentz-invariant boundary action of the confining string and its universal contribution to the inter-quark potential, JHEP 05, 130 (2012), [arXiv:1202.1984 [hep-th]].

[24] B. B. Brandt, Probing boundary-corrections to Nambu-Goto open string energy levels in 3d SU(2) gauge theory, JHEP 02, 040 (2011), [arXiv:1010.3625 [hep-lat]]. 